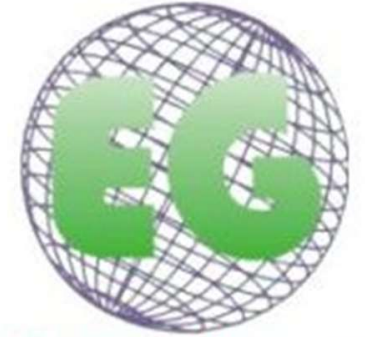

ISSN 1695-6141 N58

\title{
Miedo ante la muerte y calidad de vida en adultos mayores
}

Fear of death and quality of life in older adults

\author{
Tirso Duran-Badillo ${ }^{1}$ \\ Maria Aurelia Maldonado Vidales ${ }^{1}$ \\ Ma de la Luz Martínez Aguilar ${ }^{1}$ \\ Gustavo Gutiérrez Sánchez ${ }^{1}$ \\ Hermelinda Ávila Alpirez ${ }^{1}$ \\ San Juana López Guevara ${ }^{1}$
}

\begin{abstract}
${ }^{1}$ Unidad Académica Multidisciplinaria Matamoros-Universidad Autónoma de Tamaulipas (UAMM-UAT). México.tduran@docentes.uat.edu.mx
\end{abstract}

\section{https://doi.org/10.6018/eglobal.364291}

Recibido: 25/02/2019

Aceptado: 30/04/2019

\section{RESUMEN:}

Objetivo: Conocer la relación entre el miedo a la muerte y calidad de vida de los adultos mayores. Material y Método: Estudio correlacional en adultos mayores. La muestra fue de 99 adultos mayores, se utilizó la Escala original de Collet-Lester de Miedo a la Muerte y al Proceso de Morir y el Cuestionario WHOQOL-BREF.

Resultados: No se encontró relación en la escala global del miedo ante la muerte y el proceso de morir con la calidad de vida. Se observó relación negativa entre el miedo a la propia muerte y la dimensión física de la calidad de vida $(p<.05)$ y en el miedo al propio proceso de morir con la dimensión ambiental de la calidad de vida $(p<.05)$. Se encontró relación positiva entre el miedo a la muerte de los otros y la dimensión social de la calidad de vida $(p<.05)$.

Conclusiones: A menor miedo a la propia muerte mayor calidad de vida en la dimensión física, a menor miedo al propio proceso de morir mayor calidad de vida en la dimensión ambiental y a mayor miedo a la muerte de otras personas mayor calidad de vida en la dimensión social.

Palabras clave: Miedo; muerte; calidad de vida; anciano.

\section{ABSTRACT:}

Objective: Know the relationship between the fear of death and the quality of life of older adults.

Materials and Method: Correlational study in older adults. The sample was 99 older adults. The original Collet-Lester Scale of Death Fear and the Dying Process and the WHOQOL-BREF Questionnaire were used.

Results: No relationship found on the global scale of Death Fear and the Dying Process with the quality of life. A negative relationship was observed between the fear of own death and the physical dimension of the quality of life $(p<.05)$, and the fear of the process of dying with the environmental dimension of quality of life $(p<.05)$. Positive relationship was found between the fear of the death of others and the social dimension of quality of life $(p<.05)$. 
Conclusions: A lesser fear of one's own death higher quality of life in the physical dimension, a lesser fear of the process of dying, greater quality of life in the environmental dimension and the greater the fear of the death of other people, the greater the quality of life in the social dimension.

Key words: Fear; death; quality of life; aged.

\section{INTRODUCCIÓN}

El fenómeno del envejecimiento se encuentra presente a nivel mundial. El proceso de envejecimiento genera cambios importantes en los estilos de vida, que afectan tanto al aspecto biológico, psicológico y social(1). Los cambios que ocurren con el envejecimiento, así como los problemas físicos y/o mentales que son ocasionados por problemas de salud y experiencias de cercanía ante la muerte pueden ocasionar miedo ante la muerte y esta puede favorecer el deterioro de la calidad de vida.

Entre los cambios que se presentan con el envejecimiento se encuentran las situaciones que rondan en torno a la muerte y el proceso de morir causando miedo y confusión, ya que es una etapa en la que ha experimentado la pérdida de familiares cercanos, además de padecer enfermedades ${ }^{(2)}$.

En la etapa de adultez mayor la persona evalúa todo lo acontecido a lo largo de la vida, en donde por un lado acepta la manera como ha vivido y muestra interés para encontrar motivaciones que le permitan tener una mayor aceptación de la muerte propia o de algún familiar(3). Por el contrario, tiende a presentar emociones negativas, al saber que se encuentra en su etapa final y no se atreven hablar abiertamente sobre ello. Estas emociones tienen gran impacto en el significado que el adulto mayor tiene acerca de la muerte y sobre el proceso de morir, lo cual le provoca sufrimiento y puede alterar sus pensamientos y retrasar la aceptación de la muerte ${ }^{(4)}$.

Las experiencias de vida determinan el significado que las personas otorgan a la muerte y el proceso de morir, el cual está relacionado con el contexto en que se encuentre el adulto mayor y sus antecedentes. En este sentido cuando las personas se preparan y aceptan la muerte, pueden afrontar este proceso de una manera positiva y vivir la última etapa de su vida con mayor calidad. La calidad de vida se define como el estado de bienestar general, medido a través de las dimensiones: física, social y emocional(5).

Con base en la experiencia de los investigadores, tanto el miedo ante la muerte y el proceso de morir, como la calidad de vida, son variables que es importante sean estudiadas por el equipo multidisciplinar de salud, debido a que afectan de manera negativa en el adulto mayor y familia. En el adulto mayor el daño se refleja en el deterioro emocional y espiritual con impacto en las relaciones familiares y sociales. En la familia se presenta sufrimiento al experimentar el deterioro emocional y espiritual del adulto mayor.

En la búsqueda de literatura no se han identificado estudios en los que se analice la relación entre el miedo ante la muerte y el proceso de morir con la calidad de vida en adultos mayores. Existe poca evidencia empírica del estudio del miedo ante la muerte y el proceso de morir, en Colombia encontraron que el $63.5 \%$ de los adultos mayores resultaron con miedo ante la muerte ${ }^{(6)}$. Respecto a la calidad de vida, datos recientes de un estudio en población mexicana indican una percepción deficiente de la calidad 
de vida en el $45.4 \%$ de los adultos mayores, con mayor afección en la dimensión

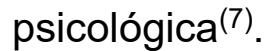

Por lo que se considera que para incrementar el cuerpo de conocimientos de enfermería es importante realizar un estudio basado en la siguiente pregunta de investigación: ¿Cómo se relaciona el miedo a la muerte y el proceso de morir con la calidad de vida de los adultos mayores? Se espera que los resultados permitan orientar el desarrollo de intervenciones centradas en redireccionar el miedo a la muerte y el proceso de morir en los adultos mayores y con ello mejorar su calidad de vida.

\section{METODOLOGÍA}

Participaron en el estudio adultos mayores de 60 años que consultan en el centro comunitario "Las culturas" perteneciente a la Secretaría de Salud de Matamoros, Tamaulipas, México. Los datos se recolectaron desde enero hasta julio del 2017. La muestra fue conformada por 99 adultos mayores de ambos sexos. Debido a que se desconoce el universo de estudio, los adultos mayores se identificaron a través de un muestreo no probabilístico en técnica de bola de nieve, que consiste en pedir al primer sujeto que identifique a otro sujeto potencial que también cumpla con los criterios de la investigación, donde los criterios de inclusión fueron tener más de 60 años, con capacidad de comunicarse, orientados en tiempo, lugar y persona, lo que se constató con preguntas como su nombre, día de la semana, mes, año y lugar donde se encontraban. Dicho muestreo se utilizó debido a que se desconoce el marco muestral, lo que imposibilitó emplear un muestreo de tipo probabilístico.

Se aplicó la Escala Miedo a la Muerte de Collett-Lester ${ }^{(8)}$, en la versión adaptada al español, con 28 ítems. Se establecieron opciones de respuesta tipo Likert que van desde mucho (6 puntos) hasta nada (1 punto), está conformada por cuatro subescalas que proporcionan información multidimensional sobre el Miedo a la Muerte propia (1, $4,6,14,17,20,23,26$ y 28), miedo a la muerte de los otros $(2,7,9,13,18,19,21$, 27,32 y 33), miedo al propio proceso de morir $(5,12,15,24,30$ y 36$)$ y el miedo al proceso de morir de los otros $(3,8,10,11,16,22,25,29,31,34$ y 35). Las respuestas de los ítems $3,6,7,8,9,10,12,14,16,18,21,23,24,25,26$ y 28 son negativas, por lo que se deben recodificar. De esta manera, la escala global tiene un puntaje máximo de 216 puntos, mayor puntuación indica mayor miedo ante la muerte. Se consideró sin miedo a los adultos mayores que resultaron con 36 puntos, algo de miedo de 37 a 144 puntos y con mucho miedo de 145 a 216 puntos.

Para evaluar la calidad de vida se utilizó la Escala WHOQOL-BREF de la Organización Mundial de la Salud ${ }^{(9)}$. Esta Escala está compuesta por 26 ítems, las dos primeras preguntas son independientes e indagan la percepción general del individuo sobre la calidad de vida y su salud respectivamente, las 24 restantes evalúan cuatro dimensiones específicas de la Calidad de Vida: Física (ítem 3, 4, 10, 15, 16, 17 y 18), Psicológica (ítem 5, 6, 7, 11, 19 y 26), Social (ítem 20, 21 y 22) y Ambiental (ítem 8, 9, 12, 13, 14, 23, 24 y 25). Cada pregunta se valora en una escala de 1 a 5 , mientras más alta sea la puntuación asignada, más alta será la calidad de vida. Se invierten los puntajes de los ítems negativos $(3,4$ y 26$)$ y los puntajes totales obtenidos se convierten en una escala de 0 a 100 para poder establecer comparaciones entre los dominios, ya que éstos están compuestos por un número desigual de reactivos. La puntuación de la percepción de la calidad de vida y la salud 
de los adultos mayores, se clasificó en deficiente cuando se encontró entre 0 y 2.99 , aceptable de 3 a 3.99 y alta entre 4 y 5 .

Los datos fueron procesados y analizados en el programa estadístico Statistical Package for the Social Sciences (SPSS) versión 21 para Windows. Se aplicó la prueba de Kolmogorov Smirnov con corrección de Lilliefors y en función de los resultados se utilizó el coeficiente de correlación de Spearman para responder el objetivo planteado. El estudio se apegó a los lineamientos establecidos en la Ley General de Salud en Materia de Investigación para la Salud(10). Se contó con el consentimiento informado de los sujetos de estudio, aprobación de las autoridades correspondientes para realizar el trabajo de campo y autorización del comité de ética e investigación, con número de registro 042.

\section{RESULTADOS}

El 59.6\% (59) de los adultos mayores que participaron en el estudio fueron del sexo femenino, con una edad promedio de 69.2 años (7.283), el 55.6\% (55) con pareja marital, el $40.4 \%$ (40) reportó tener un empleo remunerado, el $86.9 \%$ no había sido hospitalizado en los últimos seis meses, el 53.5\% (53) padece por lo menos una enfermedad crónica, el 28.3\% (28) indicó que en los últimos seis meses había muerto algún ser querido. El $27.3 \%$ (27) vive solamente con su esposo (a) y el $70.7 \%$ (70) pertenece a la religión católica (Tabla 1).

Tabla 1: Personas con las que viven y religión de los adultos mayores. Matamoros, Tamaulipas, México, 2017.

\begin{tabular}{|l|l|l|}
\hline Variable & $f$ & $\%$ \\
\hline Vive con & & \\
\hline Esposo (a) & 27 & 27.3 \\
\hline Hijos (as) & 16 & 16.2 \\
\hline Hijos (as) y familia & 14 & 14.1 \\
\hline Esposo(a) e hijos & 24 & 24.2 \\
\hline Esposo (a), hijos y familia & 4 & 4.0 \\
\hline Otros & 14 & 14.1 \\
\hline Religión & & \\
\hline Católica & 70 & 70.7 \\
\hline Cristiana & 15 & 15.2 \\
\hline Testigo de Jehová & 8 & 8.1 \\
\hline Otra & 4 & 4.0 \\
\hline Ateo & 2 & 2.0 \\
\hline
\end{tabular}

Todos los adultos mayores presentaron miedo ante la muerte y el proceso de morir. En la escala global el $87.9 \%$ (87) resultó con algo de miedo y el $12.1 \%$ (12) con mucho miedo. En el análisis por dimensiones, en el miedo a la propia muerte, el $98.0 \%$ (97) manifestó algo de miedo y el $2.0 \%$ (2) mucho miedo; en el miedo a la muerte de otros el $50.5 \%$ (50) con algo de miedo y el $49.5 \%$ (49) con mucho miedo; en el miedo al propio proceso de morir el $50.5 \%$ (50) con algo de miedo y el $49.5 \%$ (49) con mucho miedo y en el miedo al proceso de morir de otros el $87.9 \%$ (87) con algo de miedo y el $12.1 \%$ (12) con mucho miedo.

Respecto a la variable calidad de vida, el 18.2\% (18) percibió deficiente su estado de salud, el $49.5 \%$ aceptable y el $32.3 \%$ (32) alta. El 3.0\% (3) percibió deficiente su 
calidad de vida, el $64.6 \%$ (64) aceptable y el 32.3\% (32) como alta. En el análisis por dimensiones de la calidad de vida, se observó que la dimensión más afectada es la ambiental con una puntuación promedio de 52.84 ( $D E=9.72$; Tabla 2).

Tabla 2: Descripción del miedo ante la muerte y el proceso de morir y la calidad de vida de los adultos mayores. Matamoros, Tamaulipas, México, 2017.

\begin{tabular}{|l|l|l|l|l|}
\hline \multirow{2}{*}{ Variable } & \multirow{3}{*}{ Media } & \multirow{2}{*}{$\mathrm{DE}$} & \multicolumn{2}{|l|}{ IC 95\% } \\
\cline { 3 - 5 } & & & $\begin{array}{l}\text { Límite } \\
\text { inferior }\end{array}$ & $\begin{array}{l}\text { Límite } \\
\text { superior }\end{array}$ \\
\hline Edad & 69.20 & 7.28 & 67.75 & 70.62 \\
\hline Escolaridad & 6.48 & 4.28 & 5.62 & 7.31 \\
\hline Miedo a la propia muerte & 26.76 & 4.84 & 25.68 & 27.80 \\
\hline Miedo a la muerte de los otros & 40.04 & 5.36 & 39.07 & 40.97 \\
\hline Miedo al propio proceso de morir & 24.53 & 3.90 & 23.75 & 25.37 \\
\hline Miedo al proceso de morir de otros & 37.47 & 6.91 & 36.13 & 38.87 \\
\hline $\begin{array}{l}\text { Escala global de miedo ante la } \\
\text { muerte }\end{array}$ & 128.81 & 15.09 & 126.14 & 131.90 \\
\hline Calidad de vida física & 57.32 & 10.33 & 55.46 & 59.28 \\
\hline Calidad de vida psicológica & 64.94 & 10.92 & 63.44 & 67.10 \\
\hline Calidad de vida social & 56.64 & 14.43 & 54.21 & 59.87 \\
\hline Calidad de vida ambiental & 52.84 & 9.72 & 51.04 & 54.81 \\
\hline
\end{tabular}

Con la prueba $U$ de Mann Whitney solamente se observó diferencias por género en la dimensión miedo ante la muerte de los otros, donde los hombres resultaron con mayor miedo en comparación con las mujeres $(U=850.000 ; p=.018)$; así mismo, el miedo ante la muerte de los otros $(U=858.500 ; p=.021)$ y el miedo al propio proceso de morir $(U=868.500 ; p=.026)$ se presentó con mayor intensidad en los que no tienen un empleo remunerado en comparación con los que sí lo tienen.

No se observaron diferencias estadísticamente significativas en el miedo ante la muerte en los que tienen pareja marital $(p>.05)$, en los que tienen un empleo remunerado $(p>.05)$, en los que padecen enfermedades crónicas $(p>.05)$ y en los que experimentaron la muerte de un ser querido en los últimos seis meses $(p>.05)$ en comparación con los que no.

En la calidad de vida, las dimensiones en las que se encontraron diferencias significativas fueron la psicológica $(U=780.500 ; p=.004)$ y social $(U=738.000 ; p=.001)$, donde las mujeres reflejaron menor calidad de vida en comparación con los hombres. Así mismo, en las dimensiones psicológica $(U=919.000 ; p=.039)$ y social $(U=595.500$; $p=.000$ ) los que no tienen pareja marital resultaron con menor calidad de vida en comparación con los que sí la tienen. En las dimensiones física $(U=678.000 ; p=.000)$, psicológica $(U=851.000 ; p=.018)$ y social $(U=740.500 ; p=.001)$ los adultos mayores que no reportaron tener empleo remunerado, resultaron con menor calidad de vida. En la dimensión psicológica $(U=350.000 ; p=.029)$ los que habían sido hospitalizados en los últimos seis meses resultaron con menor calidad de vida. No se observaron diferencias estadísticamente significativas en la calidad de vida de los que padecen enfermedades crónicas ( $p>.05)$ y en los que experimentaron la muerte de un ser querido en los últimos seis $(p>.05)$ meses en comparación con aquellos que no.

En respuesta al objetivo general que se planteó, inicialmente se hizo un análisis correlacional del miedo ante la muerte y el proceso de morir con la calidad de vida de 
los adultos mayores y no se encontró relación estadísticamente significativa. En seguida se hizo un análisis por dimensiones de ambas variables y se observó relación negativa y significativa entre la dimensión de miedo a la propia muerte y la dimensión física de la calidad de vida $(p<.05)$ y en el miedo al propio proceso de morir con la dimensión ambiental de la calidad de vida $(p<.05)$. Por último, se encontró relación positiva entre el miedo a la muerte de los otros y la dimensión social de la calidad de vida $(p<.05$; Tabla 3$)$.

Tabla 3. Relación entre miedo ante la muerte y el proceso de morir con la calidad de vida de adultos mayores. Matamoros, Tamaulipas, México, 2017.

\begin{tabular}{|l|l|l|l|l|l|}
\hline \multirow{2}{*}{ Variables } & Física & Psicológica & Social & Ambiental & Edad \\
\cline { 2 - 6 } & $r_{\mathrm{s}}$ & $\mathrm{r}_{\mathrm{s}}$ & $\mathrm{r}_{\mathrm{s}}$ & $\mathrm{r}_{\mathrm{s}}$ & $\mathrm{r}_{\mathrm{s}}$ \\
\hline Edad & $\mathbf{- . 2 5 2 ^ { * }}$ & -.035 & $\mathbf{- 2 4 5}$ & .009 & 1 \\
\hline Miedo a la propia muerte & $-\mathbf{2 4 4 ^ { * }}$ & -.031 & -.114 & -.090 & .034 \\
\hline $\begin{array}{l}\text { Miedo a la muerte de los } \\
\text { otros }\end{array}$ & -.034 & .131 &. $\mathbf{2 5 7 ^ { * }}$ & -.029 & -.143 \\
\hline $\begin{array}{l}\text { Miedo al propio proceso de } \\
\text { morir }\end{array}$ & .003 & -.038 & .155 & $\mathbf{- . 2 0 6 ^ { * }}$ & $-.390^{* *}$ \\
\hline $\begin{array}{l}\text { Miedo al proceso de morir de } \\
\text { otros }\end{array}$ & -.137 & -.101 & -.049 & -.176 & $-.239^{*}$ \\
\hline $\begin{array}{l}\text { Escala global del miedo ante } \\
\text { la muerte }\end{array}$ & -.151 & -.030 & .051 & -.173 & $-.226^{*}$ \\
\hline
\end{tabular}

Estadísticamente significativo: ${ }^{*} p<.05 ;{ }^{* *} p<.01$

\section{DISCUSIÓN}

Este estudio permitió verificar empíricamente la relación entre las dimensiones del miedo ante la muerte y el proceso de morir con la calidad de vida. Se observó que a menor miedo a la propia muerte mayor calidad de vida en la dimensión física, esto podría deberse a que cuando el adulto mayor está consciente que se encuentra en la última etapa de vida, acepta los cambios que han ocurrido con el envejecimiento y aunque presente deterioro físico, puede no percibirse con daño debido a que se ha adaptado a los cambios y acepta su envejecimiento ${ }^{(11)}$.

Cuando el adulto mayor se encuentra en un ambiente en condiciones óptimas se siente seguro, por el contrario si el entorno en que se encuentra en su vejez es inseguro, puede presentar miedo a su propio proceso de morir, esto es evidente con lo encontrado en el presente estudio, donde se identificó que a menor miedo al propio proceso de morir mayor calidad de vida en la dimensión ambiental, al respecto, la literatura indica que el miedo al proceso de morir ocurre por la incertidumbre del lugar en que va a morir, en nuestra cultura, actualmente las familias prefieren que sea en un ambiente hospitalario, con la intención de otorgar cuidados especializados, sin pensar y tomar en cuenta la decisión del adulto mayor, quien posiblemente preferiría estar en un ambiente familiar ${ }^{(12)}$.

Cuando se trata de la muerte de los otros, la situación cambia, en este estudio se encontró que a mayor miedo a la muerte de otras personas mayor calidad de vida en la dimensión social, lo que pudiera deberse a que cuando los adultos mayores experimentan la muerte de otras personas y además saben que se encuentran próximos a morir, toman conciencia de la importancia del autocuidado y lo aplican, lo que les ayuda a mejorar su calidad de vida. En este sentido, algunos autores plantean 
que el contexto y circunstancias en que el adulto mayor se encuentra, facilitan la postura que éste tomará ante la muerte y el proceso de morir(13); es decir, de acuerdo con el contexto social y experiencia de vida del adulto mayor, la muerte de otras personas se traduce en una nueva experiencia sobre el fin de la vida del adulto mayor y esta nueva experiencia conlleva aplicación de cambios en el estilo de vida que favorecen la calidad de vida del adulto mayor.

Para plantear intervenciones de enfermería que permitan modificar el miedo ante la muerte y el proceso de morir, se considera necesario una caracterización de los sujetos que presentan mayor miedo; en este sentido en el presente estudio se identificó que los hombres resultaron con mayor miedo ante la muerte de otros en comparación con las mujeres, lo que pudiera deberse a que en nuestra cultura, las mujeres continúan cumpliendo con la expectativa social de ser cuidadoras de otros, lo que les genera satisfacción y experiencia positiva ante el envejecimiento ${ }^{(14)}$.

Por otra parte el miedo ante la muerte de los otros y el miedo al propio proceso de morir se presentó con mayor intensidad en los que no tienen un empleo remunerado en comparación con los que sí lo tienen, por lo que puede plantearse al empleo como un factor protector del miedo ante la muerte y el proceso de morir; no obstante, es una etapa de vida en la que gran parte de los adultos mayores se encuentra jubilados o incondicionados para trabajar, por lo que es necesario diseñar intervenciones de enfermería para modificar el miedo.

Se encontró que las mujeres y los que no tienen pareja marital reflejaron menor calidad de vida en la dimensión psicológica y social, contrario a lo reportado por otros autores quienes hicieron un estudio en población mexicana y en el análisis por género no encontraron diferencias en la calidad de vida(14). Los mismos autores plantean que habitualmente, las mujeres enviudan en mayor proporción que los hombres, además que cuando los hombres enviudan se vuelven a casar, a diferencia de las mujeres que prefieren quedarse solas. Sin embargo, estas variables no fueron analizadas en ambos estudios, por lo que se recomienda profundizar en ellas en futuras investigaciones.

Los adultos mayores que no reportaron tener empleo remunerado, resultaron con menor calidad de vida en la dimensión física, psicológica y social; esto explica que el trabajo, además de contribuir económicamente y cubrir las necesidades, ayuda a las personas a mantenerse activas física y socialmente, con impacto en la dimensión psicológica. Además, se piensa que el efecto negativo del cese de la actividad laboral sobre la calidad de vida de los adultos mayores, pudiera deberse a que en nuestra cultura mexicana no hay una preparación para el retiro.

En México, se busca mejorar la calidad de vida de los adultos mayores, principalmente en los de menores recursos económicos, a través de ayudas para la alimentación, asilo, alfabetización, capacitación técnica y valores humanos ${ }^{(15)}$. No obstante, y aunque en el presente estudio no se hizo un análisis de la calidad de vida de los adultos mayores que reciben ayuda de alguna organización, los resultados generales sugieren que la actividad remunerada es un factor que contribuye en el mejoramiento de la calidad de vida, por lo que se recomienda realizar investigaciones con mayor profundidad de análisis y que incluyan dichas variables.

Por último, se observó que los que habían sido hospitalizados en los últimos seis meses resultaron con menor calidad de vida en la dimensión psicológica, situación 
que se considera clara debido a que el adulto mayor se ve sometido a procedimientos y ambiente desconocidos, además que en muchas ocasiones poco se le informa sobre su estado de salud lo que le genera incertidumbre.

\section{CONCLUSIONES}

Se observó que a menor miedo a la propia muerte mayor calidad de vida en la dimensión física, a menor miedo al propio proceso de morir mayor calidad de vida en la dimensión ambiental y a mayor miedo a la muerte de los otros mayor calidad de vida en la dimensión social. En relación con el miedo ante la muerte y el proceso de morir, los hombres resultaron con mayor miedo ante la muerte de otras personas y los que no tienen empleo remunerado presentaron mayor miedo ante la muerte de los otros y al propio proceso de morir. En cuanto a la calidad de vida las mujeres y los que no tienen pareja marital reflejaron menor calidad de vida en la dimensión psicológica y social. Los adultos mayores que no reportaron tener empleo remunerado, resultaron con menor calidad de vida en la dimensión física, psicológica y social; y los que habían sido hospitalizados en los últimos seis meses resultaron con menor calidad de vida en la dimensión psicológica.

\section{REFERENCIAS}

1. Organización Mundial de la Salud. Salud mental: fortalecer nuestra respuesta. 2016. Disponible en: http://www.who.int/mediacentre/factsheets/fs220/es

2. Urzúa A, Bravo M, Ogalde M, Vargas C. Factores vinculados a la calidad de vida en la adultez mayor. Revista médica. 2011; 139(8): 1006-1014. http://dx.doi.org/10.4067/S0034-98872011000800005

3. Alvarado AM, Salazar AM. Análisis del concepto de envejecimiento. Gerokomos. 2014; 5(2): 57-62.

4. Viel S. La experiencia emocional de envejecer. Temas de Psicoanálisis. Enero 2019; 17. Disponible en http://www.temasdepsicoanalisis.org/wpcontent/uploads/2019/01/Silvia-Viel.-Experiencia-emocional-de-envejecer.-1.pdf

5. Higuita LF, Cardona JA. Concepto de calidad de vida en la adolescencia: una revisión crítica de la literatura. Revista CES Psicología. 2015; 8(1): 155-168.

6. Uribe AF, Valderrama L, López $S$. Actitud y miedo ante la muerte en adultos

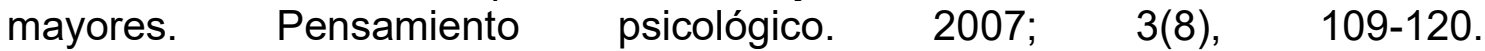
https://www.redalyc.org/pdf/801/80130809.pdf

7. Flores-Herrera BI, Castillo-Muraira Y, Ponce-Martínez D, Miranda-Posadas C, Peralta-Cerda EG, Duran-Badillo T. Percepción de los adultos mayores acerca de su calidad de vida. Una perspectiva desde su contexto familiar. Rev Enferm Inst Mex Seguro Soc. 2018; 26(2): 83-88. http://www.medigraphic.com/pdfs/enfermeriaimss/eim-2018/eim182d.pdf

8. Lester D. Escala de Miedo a la Muerte de Collett-Lester. En Neimeyer RA. Métodos de evaluación de la ansiedad ante la muerte (pp. 57-72). España: Paidós, 1997.

9. OMS. WHOQOL User Manual. Geneva, World Health Organization. 1998. En Skevington, SM, Lofty M, O'Connell KA. The World Health Organization's WHOQOL-BREF quality of life assessment: Psychometric properties and results of the international field trial A Report from the WHOQOL Groupq. Quality of LifeResearch. 2004; 13, 299-310. https://www.ncbi.nlm.nih.gov/pubmed/15085902 
10. Secretaría de salud. Reglamento de la Ley general de Salud en Materia de Investigación, México, $1987 . \quad$ Disponible en http://www.dof.gob.mx/nota detalle.php?codigo $=5339162 \&$ fecha $=02 / 04 / 2014$

11. Duran-Badillo T, Domínguez-Chávez CJ, Hernández-Cortés PL, Félix-Alemán $A$, Cruz-Quevedo JE, Alonso-Castillo MM. Dejar de ser hacer: significado de dependencia funcional para el adulto mayor. Acta Universitaria. 2018; 28 (3): 40-46. doi: 10.15174/au.2018.1614

12. Eliopoulos C. Enfermería gerontológica. China: Wolters Kluwer, 2014.

13. Olivi A, Fadda-Cori G, Pizzi-Kirschbaum M. Evaluación de la calidad de vida de los adultos mayores en la ciudad de Valparaíso. Papeles de Población. 2015; 84: 227-249.

14. González-Celis AL, Araujo AV. Estrategias de afrontamiento ante la muerte y calidad de vida en adultos mayores mexicanos. Revista Kairós Gerontología. 2010; 13(1): 167-190. https://revistas.pucsp.br/index.php/kairos/article/viewFile/4868/3450

15. Cordera R, Provencio E. Propuestas estratégicas para el desarrollo 2019-2024 (Colección: Informe del Desarrollo en México). México: Universidad Nacional Autónoma de México, 2018. Disponible en http://www.pued.unam.mx/export/sites/default/publicaciones/16/Propuestas es trategicas.pdf 